\title{
La educación superior en España en 2020: retos para alcanzar la igualdad de oportunidades*
}

Higher education in Spain in 2020: challenges to achieve equal opportunities

María Luisa De Torres Soto ${ }^{1 \star}$

Fecha correspondencia:

Recibido: 20 de abril de 2020.

Revisión: 1 de mayo de 2020.

Aceptado: 14 de julio de 2020.

Forma de citar:

De Torres - Soto, María Luisa.

"La educación superior en España

en 2020: retos para alcanzar la

igualdad de oportunidades." En:

Revista CES Derecho. Vol. 11, №. 2 ,

junio a diciembre de 2020, 160-178

Open access

Términos de uso

Licencia creative commons

Etica de publicaciones

Revisión por pares

Gestión por Open Journal System

DOl: http://dx.doi.org/10.21615/

cesder.11.2.9

ISSN: 2145-7719

Sobre el artículo:

Este estudio jurídico trae causa

de la Ponencia que pronuncié

en las I Jornadas de Enseñanza

Militar y Orientación el 24/6/2019

en el Ministerio de Defensa en

España y está realizado con los

datos publicados por organismos

oficiales actualizados a fecha

24/6/2019

Sobre la autora:

1. Universidad Alfonso X El Sabio.

\section{Resumen}

El Espacio Europeo de Educación Superior (EEES) en consonancia con los arts. 9 y 14 de la Constitución española y la actual Ley Orgánica 3/2007, de 22 de marzo, para la igualdad efectiva de mujeres y hombres (LOIEMH) ha avanzado en el objetivo de incluir más medios que permitan ofrecer en la práctica las mismas oportunidades entre mujeres y hombres. En la Universidad se han llevado a cabo numerosos esfuerzos consistentes fundamentalmente en la transmisión de valores esenciales y transversales en los planes de estudio y la creación de estructuras organizativas que promuevan el respeto y la igualdad de derechos y oportunidades. El principal reto pendiente se encuentra en la dificultad aún existente para el acceso efectivo a puestos de alta dirección y gobierno de las mujeres. La superación de esta segregación vertical requiere todavía importantes avances para revertir el denominado efecto "leaky pipeline".

Palabras claves: Igualdad, Universidad, oportunidades, responsabilidad, conciliación.

\section{Abstract}

The European Higher Education Area (EHEA) in accordance with arts. 9 and 14 of the Spanish Constitution and the current Organic Law 3/2007, of March 22, for the effective equality of women and men (LOIEMH) has advanced in the objective of including more means that allow offering in practice the same opportunities between women and men. At the University, numerous efforts have been carried out, consisting mainly of the transmission of essential and transversal values in the study plans and the creation of organizational structures that promote respect and equal rights and opportunities. The main pending challenge lies in the still existing difficulty for effective access for women to senior management and government positions. Overcoming this vertical segregation still requires significant progress to reverse the so-called "leaky pipeline" effect.

Keywords: Equality, University, opportunities, responsibility, conciliation. 
“...la máxima participación de la mujer, en igualdad de condiciones con el hombre, en todos los campos, es indispensable para el desarrollo pleno y completo de un país, el bienestar del mundo y la causa de la paz." CONVENCIÓN SOBRE LA ELIMINACIÓN DE TODAS LAS FORMAS DE DISCRIMINACIÓN CONTRA LA MUJER (Asamblea General de Naciones Unidas. Fecha: 18 de diciembre de 1979)

\section{Introducción: situación actual}

La Universidad, como Institución impulsora de la creación y difusión del conocimiento ${ }^{1}$ ha desempeñado en los últimos años un papel relevante en la eliminación de las desigualdades de género en la sociedad ${ }^{2}$, haciéndose más presente el deber de promover una igualdad real, sin perjuicio de las barreras voluntarias que siempre hay que salvar porque hay que respetar la libertad de las personas.

Con la Convención para la Eliminación de todas las Formas de Discriminación (CEDAW) por Naciones Unidas en 1979, ratificada por España en 1983, el Tratado de Ámsterdam, y el correspondiente desarrollo normativo español y comunitario, se hizo necesaria una formación para la igualdad y con ella la asunción de responsabilidades por parte de las universidades. Sin embargo, está siendo en estos últimos años, a pesar de las muchas políticas de igualdad existentes hasta el momento, cuando se está tomando verdadera conciencia de ello al verse ya casi de manera normalizada la presencia de mujeres en todos los ámbitos universitarios: estudiantes, docentes, investigadores, personal de administración y servicios (PAS) y órganos de Gobierno (AA.VV, 2018).

El Espacio Europeo de Educación Superior (EEES) está en consonancia con los arts. 9 y 14 de la Constitución española que proclaman la obligación de garantizar la igualdad por parte de los poderes públicos, así como el derecho a la igualdad y a la no discriminación por razón de sexo ${ }^{3}$. Por otra parte, la actual Ley Orgánica 3/2007, de 22 de marzo, para la igualdad efectiva de mujeres y hombres (LOIE) ha avanzado en el objetivo de incluir medios que permitan ofrecer en la práctica las mismas oportunidades. Desde la Universidad se han llevado a cabo numerosos esfuerzos en la aplicación de políticas de igualdad de oportunidades, consistentes fundamentalmente en la transmisión esencial de valores, planes de estudio y creación de estructuras organizativas, procurando conseguir en todas ellas la elaboración de instrumentos que promuevan el respeto y la igualdad de derechos y oportunidades entre mujeres y hombres.

En la actualidad, puede decirse que hemos alcanzado un logro legal y formal gracias a que contamos con numerosa normativa internacional, europea y española (estatal y autonómica) que incorpora las políticas de género4

1. "La igualdad de género se considera uno de los principios fundamentales de cualquier democracia, así como un facilitador del crecimiento económico y del bienestar social". VI Informe Conferencia Internacional del Trabajo, 98.a reunión, 2009.

2. Vid. Preámbulo de la Ley 4/2007, de 12 de abril por la que se modifica la Ley Orgánica 6/2001, de 21 de diciembre de Universidades (LOM-LOU).

3. El principio de igualdad ante la ley representa un límite frente al legislador para que las normas no creen situaciones discriminatorias o injustificadamente desiguales (STC 103/1983, de 22 de noviembre).

4. Libro Verde "El Espacio europeo de Investigación: nuevas perspectivas": la Resolución 4712 sobre incorporación de una perspectiva de género en todas las políticas y programas del sistema de las Naciones Unidas, Reglamento (UE) n 1291/2013 por el que se establece el programa H2020; la Constitución española; el Plan estratégico de igualdad de oportunidades 2018-2021; La Ley Orgánica 3/2007, de 22 de marzo de 2007, para la Igualdad efectiva entre mujeres y hombres (LOIE); la Ley Orgánica 1/2004, de 28 de diciembre, de medidas de protección integral contra la violencia de género (LOMPI) ; la Ley Orgánica 6/2001, de 21 de diciembre, de Universidades (LOU); Ley Orgánica 4/2007, de 12 de abril, por la que se modifica la Ley Orgánica 6/2001, de 21 de diciembre, de Universidades (LOM-LOU): el Real Decreto 1393/2007, de 29 de octubre, por el que se establece la Ordenación de las Enseñanzas Universitarias Oficiales; la Ley 4/2011 de 1 de junio, de Ciencia, Tecnología e Innovación (LCTI); y, el Real Decreto-ley 3/2019, de 8 de febrero, de medidas urgentes en el ámbito de la Ciencia, la Tecnología, la Innovación y la Universidad. 


\section{Los inicios de la mujer en la Universidad española}

\section{Antecedentes}

En los primeros treinta años del siglo XX (MONTERO, 2010) algunas personalidades femeninas notables, aunque sin estudios superiores, dejaron su huella en la vida social, como Federica Montseny ministra de Sanidad durante la Segunda República, Carmen de Burgos periodista; escritoras y poetas como Concha Espina, Emilia Pardo Bazán, Concha Méndez o Ernestina de Champourcin; pintoras como María Mallo, pedagogas como Concepción Sainz Otero, o damas tan cultas como Zenobia Camprubí, la esposa de Juan Ramón Jiménez. Todas ellas realizaron aportaciones de relieve en su entorno, a pesar de no haber cursado estudios universitarios. Sin embargo era un número de mujeres muy reducido.

\section{Comienzos}

La llegada de la mujer a la Universidad en 1910 coincidió con una mejora en el desarrollo del país, aunque España fue de los países que más tarde admitió a mujeres en la Universidad, incluso más tarde que el Imperio austro húngaro (1897) o que el Imperio alemán (1908). En 1910 el nivel de analfabetismo femenino era del 65,8\% (CAPEL, 1982), pues la función principal y "casi exclusiva" de la mujer era y así lo entendía la mayor parte de la sociedad, cuidar el matrimonio y educar a los hijos. "Esas ocupaciones del ámbito privado se entendían tan absorbentes que impedían cualquier otra ocupación con dimensión social a la que pudiera dedicarse la mujer" (MANGINI,2001). Otro argumento en contra con el que se encontraba la mujer para la incorporación a la Universidad era que restaba puestos de trabajo al varón (EZQUERRA, 1926). A pesar de ello, de 1871 a 1910 la lucha hasta conseguir la Educación Superior para la mujer tuvo como resultado que 77 mujeres lo consiguieron. De ellas, 53 lograron terminar sus estudios y obtuvieron el título de licenciadas o doctoras ${ }^{5}$ (FLECHA GARCÍA, 1996). Ejemplos de grandes mujeres universitarias de esos años fueron Victoria Kent, Clara Campoamor, que entre otras ocupaciones laborales ${ }^{6}$ tenían bufete abierto en Madrid ya en 1925; otras como María de Maeztu y María Zambrano también fueron mujeres insignes. Todas ellas no tenían hijos aunque algunas estuviesen casadas, cuestión que en esa época les favoreció. Las que sí además de estar casadas tenían hijos, sin embargo no llegaban a triunfar como merecían, pues el éxito, casi en exclusiva se lo llevaba el marido como ocurrió por esa época con María Goyri, doctora en Filosofía y Letras y profesora del Instituto-Escuela mujer de Menéndez Pidal (MONTERO, 2010)7.

\section{Ámbito internacional}

En el ámbito internacional, otras mujeres que han sido grandes investigadoras han sido Marie Curie (1867-1934) que descubrió el Radio y el Polonio; Tu Youyou (1930) la cura contra la malaria; Ada Lovelace (1818-1862) la programación; Rosalin Franklin (1920-1958) la estructura del ADN; Hedy Lamarr (1914-2000) el sistema de comunicación base del WIFI, GPS y Bluetooth; Susan Jocelyn Bell (Burnell) 1943, los púlsares, Vera Rubin (1928-2016) descubrió la materia oscura, etc... Aun así, en la Conferencia Solvay de 1927 había veintinueve científicos: de ellos, veintiocho hombres y una sola mujer (Marie Curie) y en 2016 en la misma Conferencia, de los cincuenta

5. Sobre el período 1871-1910 existe un riguroso y documentado trabajo por parte de esta autora.

6. La primera era una de las responsables del Instituto-Escuela y fue la que más alto logró llegar en la vida política española (Directora General de Prisiones); y la segunda tenía una plaza por oposición en el Ministerio de Instrucción Pública, dedicándose a las clases para adultas, además de su colaboración periodística.

7. Con el que se dedicó a investigar, pero apareciendo solamente la firma de él en sus escritos. 
científicos presentes, había cuarenta y dos hombres y ocho mujeres (16\%), comprobándose como aún queda mucho camino por recorrer ${ }^{8}$.

\section{Actualidad española}

Hasta el curso académico 1977-1978 no se llegó en España a los niveles de estudiantes universitarias que tenían ya los Estados Unidos en 1920: un 43\% (SALOMÓN. 1985). Solo a partir de los años setenta se aceleró realmente el movimiento de acceso femenino a la Universidad. Y como consecuencia, empezaron a desarrollarse también todos los fenómenos asociados: la incorporación de la mujer al mundo laboral, el desarrollo de carreras profesionales femeninas, los problemas de compatibilizar trabajo y familia y la legislación pertinente para hacerlo posible.

En la actualidad puede decirse que hemos alcanzado un logro legal/formal, pues aunque siempre habrá nuevas medidas a legislar, hemos avanzado desde que Elena

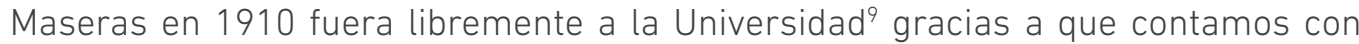
numerosa normativa internacional, europea y española (estatal y autonómica) que incorpora las políticas de género, como puede observarse con el Libro Verde "El Espacio europeo de Investigación: nuevas perspectivas"; la Resolución 4712 sobre incorporación de una perspectiva de género en todas las políticas y programas del sistema de las Naciones Unidas, Reglamento (UE) n 1291/2013 por el que se establece el programa H2020; la Constitución española; el Plan estratégico de igualdad de oportunidades 2018-2021; La Ley Orgánica 3/2007, de 22 de marzo de 2007, para la Igualdad efectiva entre mujeres y hombres (LOIEMH); la Ley Orgánica 1/2004, de 28 de diciembre, de medidas de protección integral contra la violencia de género (LOMPI); la Ley Orgánica 6/2001, de 21 de diciembre, de Universidades; Ley Orgánica 4/2007, de 12 de abril, por la que se modifica la Ley Orgánica 6/2001, de 21 de diciembre, de Universidades (LOM-LOU, 2007); el Real Decreto 1393/2007, de 29 de octubre, por el que se establece la Ordenación de las Enseñanzas Universitarias Oficiales; la Ley 4/2011 de 1 de junio, de Ciencia, Tecnología e Innovación; y, el Real Decreto-ley 3/2019, de 8 de febrero, de medidas urgentes en el ámbito de la Ciencia, la Tecnología, la Innovación y la Universidad.

Concretamente, es en el ámbito de la enseñanza militar ${ }^{10}$, la igualdad se ha alcanzado con base en la normativa citada y de manera específica con el Real Decreto-ley 1/1988, de 22 de febrero, por el que se reguló la incorporación por primera vez de la mujer a las Fuerzas Armadas (FAS) españolas. Desde entonces, el ámbito castrense ha sido ejemplo de institución impulsora en la igualdad de oportunidades con toda su normativa, gracias entre otros al apoyo del Observatorio Militar para la Igualdad entre mujeres y hombres, en los procesos de selección, formación, evaluaciones, ascensos, movilidad y destinos, en los que se ha tenido en cuenta de manera especial a) la conciliación de la vida laboral y familiar, en particular en las situaciones de embarazo, y b) la protección frente a la violencia de género, acoso sexual y abuso de poder por razón de género. El Ministerio de Defensa no cesa en la continua creación

8. Datos ofrecidos por la Unidad de Igualdad de la Subsecretaría ya indicada.

9. Elena Maseras (Facultad de Medicina de la Universidad de Barcelona 1872). Sólo a partir del 8 de marzo de 1910, la mujer fue libre para matricularse pues hasta entonces se necesitaba permiso especial del Consejo de Ministros.

10. Se cita la enseñanza en las FAS pues trae su causa en que los estudios de las Fuerzas Armadas están integrados en los estudios universitarios a través de la Red de Centros Universitarios de la Defensa (CUD), creados por R.D. 1723/2008 de 24 de Octubre. A la luz de la normativa de las FAS indicada en el texto, el ámbito castrense ha sido ejemplo de institución impulsora en la igualdad de oportunidades sin cesar en la continua creación de medidas para el fomento de la igualdad que ya con vistas al próximo marco estratégico del Ministerio de Defensa en el ámbito del Fondo Social Europeo $2021-2027$ se están estudiando. 
de medidas para el fomento de la igualdad que ya con vistas al próximo marco estratégico del Ministerio de Defensa en el ámbito del Fondo Social Europeo 2021-2027 se están estudiando.

\section{Segregación horizontal y vertical de la mujer en la Universidad}

A pesar de la puesta en marcha de políticas y programas de género, no se ha alcanzado todavía el logro material, es decir, una igualdad real y efectiva a todos los niveles, pues se observa una desventaja tanto el número de matrículas según el tipo de estudios universitarios de Grados y Postgrados (segregación horizontal) ${ }^{11}$, como en puestos de dirección y gobierno presentan diferencias entre hombres y mujeres (segregación vertical) ${ }^{12}$.

El nivel de formación alcanzado hasta la fecha es similar hasta la educación secundaria, pero ligeramente más bajo cuando se trata de estudios universitarios ${ }^{13}$.

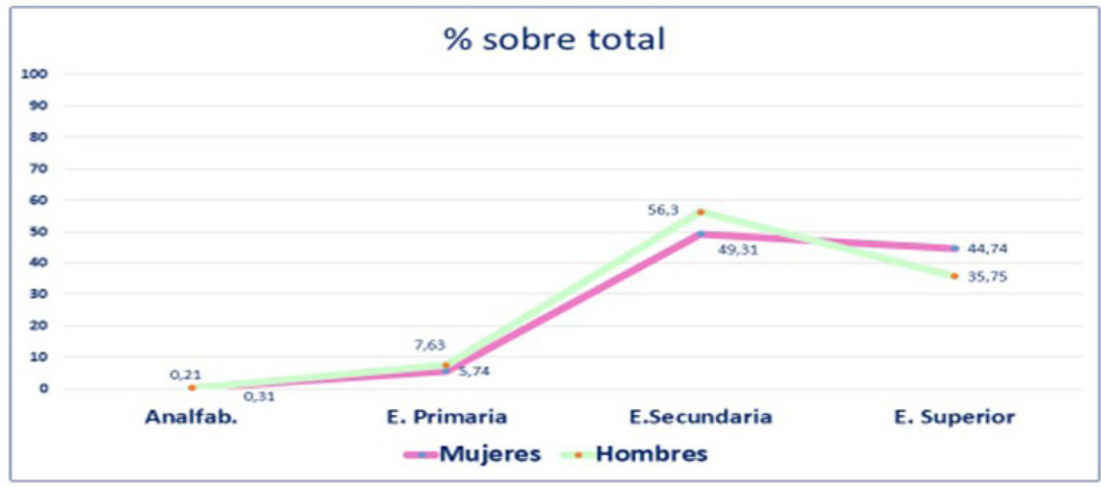

Figura 1. Población activa por nivel de formación alcanzado Curso 2017/2018.

Fuente: Datos y cifras del Sistema Universitario Español - Publicación 2018-2019 - Ministerio de Ciencia, Innovación y Universidades.

\section{Segregación horizontal: estadísticas y posibles causas}

El primer supuesto, elección de estudios -segregación horizontal-, comprobamos que se gradúan más mujeres, obtienen mejores tasas de rendimiento, éxito y evaluación y graduación en todas las etapas de Grado y Postgrado, pero las tasas de abandono en niveles Postgrado son mayores. Como muestra, según los últimos datos publicados en la web de la Unidad de Igualdad de la Subsecretaría de Ciencia, Innovación y Universidades del Ministerio de Ciencia, Innovación y Universidades en Junio de 2019 (datos del curso 2017/2018) en Grado, son 711.415 (55,1\%) mujeres las matriculadas frente a 579.729 hombres, en Máster 112.293 mujeres (49,8\%) frente a 92.576 hombres y en estudios de Doctorado 39.500 frente a 39.886 hombres. Estas cifras son semejantes en el nivel académico de Máster y Doctorado. La principal diferencia se encuentra en los estudios elegidos, pues las mujeres cursan principalmente carreras de artes y humanidades, ciencias sociales y jurídicas, y ciencias de

11. Conviene resaltar que Las notas medias en Grado Universitario, durante el curso 2017/2018 fue de 7,34 en mujeres y 7,05 en hombres.

12. En este trabajo se encuentran las estadísticas del curso 2017/2018 disponibles en la web de la Unidad de Igualdad de la Subsecretaría de Ciencia, Innovación y Universidades del Ministerio de Ciencia, Innovación y Universidades, pues el curso 2018/2019 no había finalizado en el momento de la ponencia. 
la salud tanto en Grado, como en Master y Doctorado y los hombres más en STEM (Ciencia, Tecnología, Ingeniería y Matemáticas) y TIC (Tecnología de la Información y Comunicación) ${ }^{14}$.

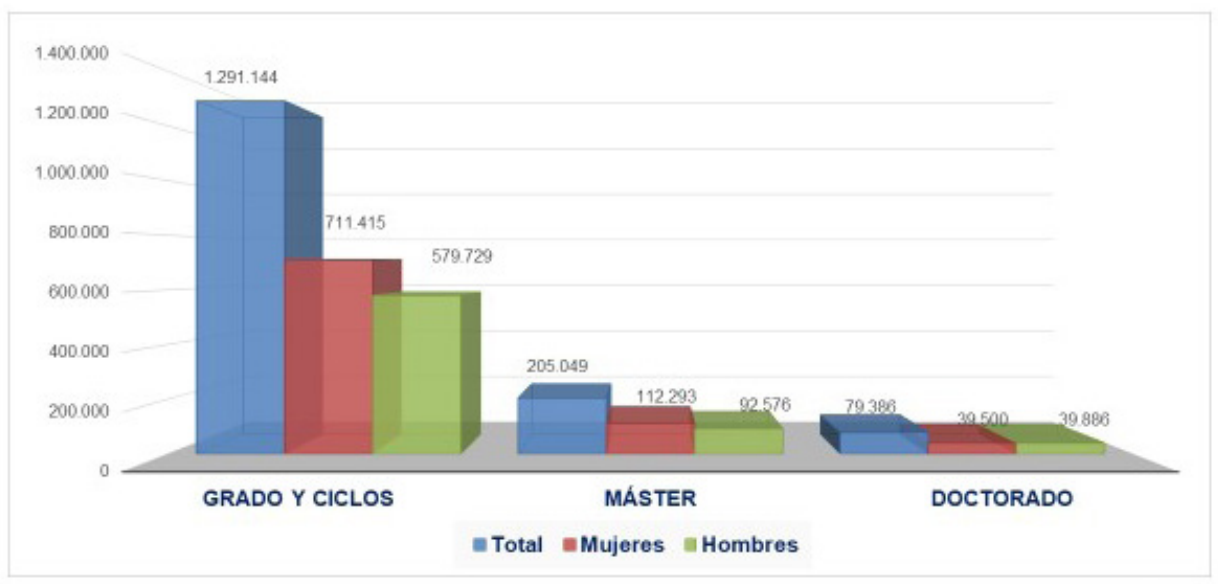

Figura 2. Tasas de abandono, idoneidad y graduación en el Grado universitario. Curso 2017/2018.

Fuente: Datos y cifras del Sistema Universitario Español - Publicación 2018-2019 - Ministerio de Ciencia, Innovación y Universidades.

No existen hasta la fecha estudios científicos oficiales sobre las causas que dan lugar a dicha segregación, aunque pueden destacarse como causas de la segregación en número de matriculaciones de Grado y Postgrado los estereotipos ya creados en el entorno social, familiar, escuela, amigos y medios de comunicación, pues el rol femenino ha estado, y de alguna manera sigue vinculado actualmente a determinadas profesiones y actividades. Y, el motivo principal del menor número de matrículas en estudios STEM Y TIC es la tradicional vinculación de estudios técnicos de Grado al personal masculino. Otro motivo relevante se centra en la falta de estudios de género en pre-grado, de manera transversal, que ayuden a romper estereotipos y faciliten la orientación vocacional pues ni en los libros de texto ni en los medios de comunicación se habla suficientemente de mujeres científicas. Por último, cabe destacar que los ejemplos de profesionales triunfadores que se dan a conocer en áreas STEM son todavía en su mayoría varones.

14. Datos disponibles en la Unidad de Igualdad del Ministerio de Ciencia, Universidad e Innovación a fecha 23-6-19. http://www.ciencia "Datos y cifras Ministerio de Ciencia.."qob.es/stfls/MICINN/Universidades/Ficheros/Estadisticas/datos-y-cifras-SUE-2018-19.pdf.

Sin que este estudio esté centrado en la incorporación de la mujer a las Fuerzas Armadas (FAS) españolas, merece la pena destacar y es de justicia recordar que las FAS son un ejemplo de interdisciplinariedad en materia de género también en el área educativa. Precisamente, en 1988, la primera mujer que ingresó en la primera promoción que admitió mujeres en las FAS españolas lo hizo en STEM, pues es Ingeniero. 


\section{ANECA}

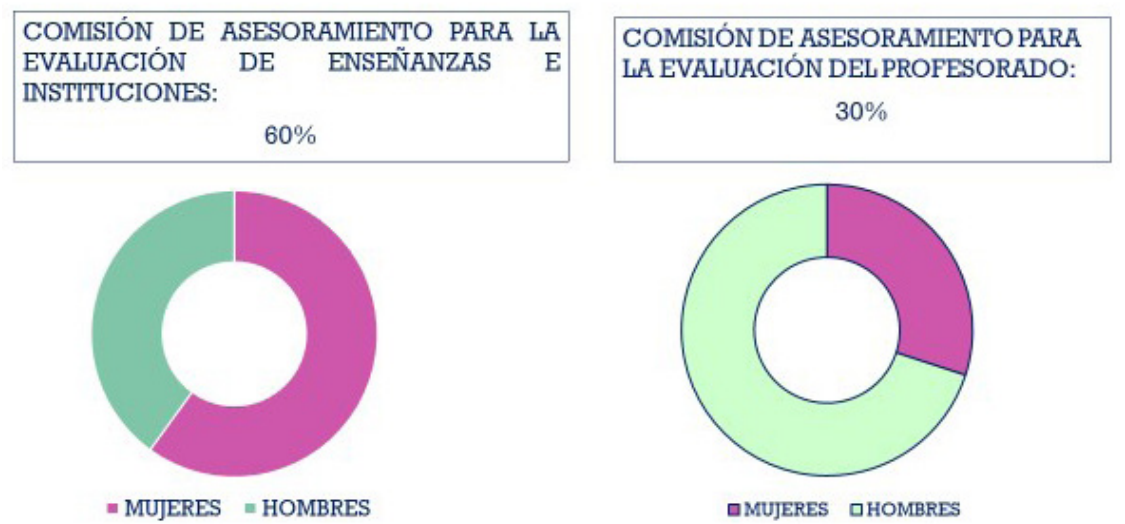

Figura 3. Número de hombres y mujeres que forman parte de las Comisiones ANECA. Curso 2017/2018.

\section{Segregación vertical: estadísticas y posibles causas}

En cuanto a la falta de igualdad en el segundo supuesto, es decir, en los puestos de investigación y de dirección -segregación vertical-, se puede hablar de un glass ceiling o "techo de cristal" esto es, una barrera invisible que dificulta a las mujeres acceder a niveles de responsabilidad y decisión ${ }^{15}$ : por ejemplo, hay más mujeres docentes y pocas catedráticas, una situación que está generalizada en Europa.

Como curiosidad, desde el punto de vista internacional, de los 609 Premios Nobel concedidos entre 1901 y 2018, solo 18 han sido concedidos a mujeres. En el ámbito español, en puestos de liderazgo como es la Dirección de Organismos Públicos de Investigación (OPI,s) ${ }^{16}$ las mujeres representan el 37,5\% del total; y, asimismo, en la Agencia Nacional de Evaluación de la Calidad y Acreditación (ANECA), la presencia de mujeres es del $60 \%$ en la Comisión de asesoramiento para la evaluación de enseñanzas e instituciones, a diferencia de la Comisión de asesoramiento para la evaluación del profesorado en que sólo ostentan el 30\% del total del personal. En cuanto a puestos de gobierno y liderazgo, a día de hoy, 24 de junio de 2019, son nueve las mujeres Rectores en Universidades Públicas, representando el 21\% del total.

Los motivos estereotipados que se vinculan fundamentalmente a esta segregación vertical hasta el momento, se centran en: a) los pocos conocimientos en estudios de género pues faltan Grados y Postgrados específicos y su transversalidad ${ }^{17}$; b) la falta de mujeres en estudios de liderazgo; c) la falta de conocimiento de los resultados estadísticos en género que no son de fácil acceso al público en general; e) la falta de conocimiento de los nombres y apellidos de mujeres investigadoras; f) la falta de dotación económica para políticas de género; g) la falta de nuevas medidas de conciliación; y, h) la falta de denuncias por violencia de género.

15. Vid. DE PABLO, Flora "las científicas y el techo de cristal" en http: www.amit-es.org/ entre otros.

16. Estadística sobre actividades de I+D 2017 (prov.) y EPA $4^{\circ} \mathrm{T}$ 2018. INE.

17. En la actualidad existen 30 Master universitarios oficiales en materia de igualdad y violencia de género, estudios de género y políticas de igualdad, que pueden consultarse en el Registro de Universidades, Centros y Títulos del Ministerio de Ciencia, Investigación y Universidades (Conferencia de Rectores 2018) 
Por todo lo expuesto y en especial en las ramas científicas se puede afirmar que la presencia de mujeres en la Universidad y en I+D+i está presidida por la teoría de "Leaky pipeline" o "tubería que gotea", término creado por la UNESCO, pues en España, las mujeres van abandonando su carrera profesional con más frecuencia que los hombres conforme van progresando en ella. Así, las estudiantes de Grado representan el 54,7\%, las de Máster el 54,4\%, las de Doctorado 49,6\% y las científicas el $39,1 \%{ }^{18}$.

\section{Leaky
pipeline
(tuberia
que gotea)}

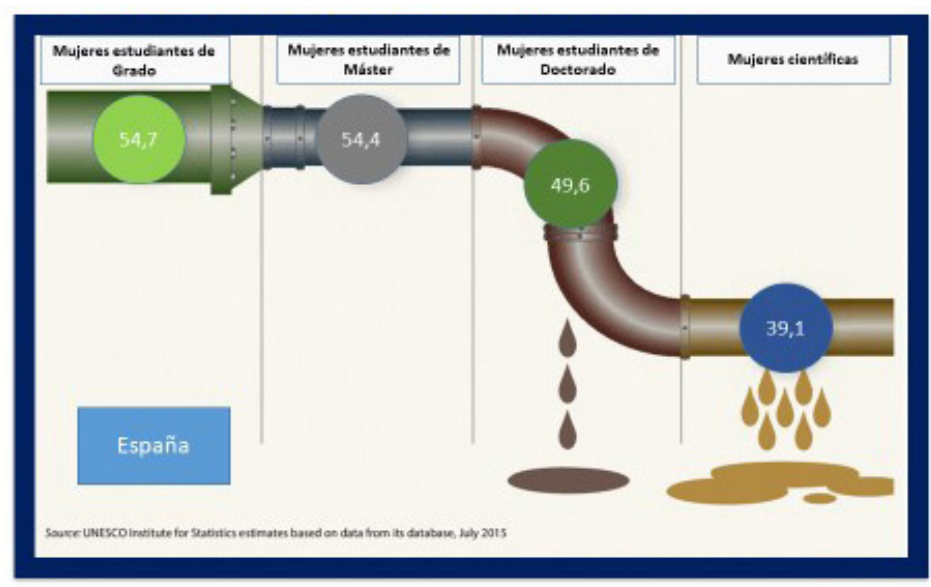

Figura 4. Presencia de mujeres en I+D+i. Curso 2017/2018.

A todas las áreas profesionales que se han indicado debe sumarse un factor prioritario y casi podríamos decir el más común y de mayor interés, que es la ausencia de nuevas medidas de conciliación personal, familiar y laboral. Hay que recordar que, hasta el momento son las mujeres, las que en la mayoría de los casos, siguen solicitando permisos de conciliación para la atención de familiares a cargo, y, como consecuencia de ello, ante la ausencia laboral durante años, el problema que se encuentran las mujeres para ocupar puestos de dirección y gobierno es mucho mayor (también es un motivo de "leaky pipeline")

En cuanto al Personal de Administración y Servicios (PAS) en la Universidad, las mujeres ocupan el $61 \%$ del total, pues del total de 61.908, las mujeres ocupan 37.657, entre personal funcionario, contratado y eventual. Se observa que hay más contratadas cuando el nivel de estudios es inferior.

\section{Igualdad de oportunidades en la Universidad}

La LOIE en el ámbito de la Educación Superior, recoge que "las Administraciones fomentarán la enseñanza y la investigación sobre el significado y alcance de la igualdad entre mujeres y hombres (art. 25.1), centrándose en "a) La inclusión, en los planes de estudio en que proceda, de enseñanzas en materia de igualdad entre mujeres y hombres. b) La creación de Postgrados específicos. c) La realización de estudios e investigaciones especializadas en la materia" (art. 25.2). Por su parte, el art. 4 de la LOMPI prescribe que "Las Universidades incluirán y fomentarán en todos los ámbitos académicos la formación, docencia e investigación en igualdad de género y no discriminación de forma transversal". Ello es debido a que la política de género es la aplicación del concepto mainstreaming o "transversalidad de género" en los diferentes ámbitos de intervención de las políticas públicas y de la igualdad de oportunidades entre mujeres y hombres (art. 15 LOIEMH) ${ }^{19}$.

18. Merece la pena destacar que, en el ámbito castrense, la segregación vertical es menor, pues las FAS españolas han sido las que más rápidamente han hecho posible que las mujeres lleguen a los empleos que actualmente ostentan. 


\section{Medidas actuales de garantía}

Para conseguir los fines indicados en el apartado anterior, a corto y medio plazo se cuenta con varios instrumentos:

En primer lugar debe destacarse el Programa/Estrategia Horizonte H2020 Reglamento (UE) 1291/2013, que tiene como fin la inclusión de la materia de género como parte de los costes subvencionables del proyecto. Actualmente, España está por encima de la Unión Europea pues tiene un 33\% de paridad de mujeres y hombres en los comités científicos y administrativos, mientras que la media en Europa está en 28\%. La Estrategia H2020 pretende alcanzar la cuota 40\%-60\% de presencia de hombres y mujeres en el ámbito profesional ${ }^{20}$. Y esta es la única cuota regulada que existe, debiéndose aplicar de forma que, a su vez, no cree nuevas discriminaciones ${ }^{21}$.

El II Plan para la Igualdad entre mujeres y hombres en la Administración General del Estado y sus organismos públicos, que es aplicable también al ámbito universitario, está orientado a cumplir las líneas del Plan Estratégico de Igualdad de Oportunidades 2014-2016. Por ello, se centra en la igualdad en el empleo, la conciliación y corresponsabilidad y la lucha contra la violencia de género, movilidad de la víctima sin reducción en la retribución, uso del lenguaje inclusivo ${ }^{22}$, fomento de la creación de guías de conciliación en universidades y OPI's, y el establecimiento de buzones de sugerencias, entre sus objetivos prioritarios.

En este sentido, como Medidas de conciliación, el personal de investigación en situación de incapacidad temporal, riesgo durante el embarazo, lactancia, maternidad, paternidad, adopción o acogimiento, tendrá garantizadas las mismas oportunidades en los procesos de selección y evaluación y su expediente no será penalizado por el tiempo transcurrido en estas situaciones (arts. 64 y 76 LOM-LOU). Por otra parte y, en el caso del nacimiento de un hijo, las 6 primeras semanas de baja son obligatorias e intransferibles para la mujer y no así las 10 siguientes. Para el hombre existe un permiso de las 5 primeras semanas voluntarias e intransferibles. En este sentido, la Ley de Presupuestos Generales del Estado de 2019 y la Proposición de Ley en el Congreso han recogido la ampliación a 16 semanas de los permisos de paternidad ${ }^{23}$. Además, deben impulsarse políticas y actuaciones que consideren el tiempo de la maternidad y cuidado de los hijos en el curriculum de las investigadoras ${ }^{24}$. Sería interesante también crear ayudas para pagar a cuidadores cuando hay que asistir a Congresos Internacionales; publicar una guía de conciliación en las Universidades; respetar horarios de reuniones en horas centrales del día; fomentar el teletrabajo y crear guarderías en lugares de trabajo. Algunas de estas medidas son impulsadas por la normativa indicada, aunque aún no se han hecho efectivas.

por lo que las administraciones públicas lo deben integrar, de forma activa, en la adopción y ejecución de sus disposiciones normativas, en la definición y presupuestación de políticas públicas en todos los ámbitos y en el desarrollo del conjunto de todas sus actividades".

20. Así lo recoge también la LOIE - D.A. $n^{\circ} 1$.

21. La LOU en el mismo sentido establece la "presencia equilibrada" (órganos de gobierno (art. 13), directores de departamento (art. 25), y equipos de investigación (art. 41.4), y, por aplicación también de la LOEI, en caso de que no pueda darse dicho equilibrio, deberá motivarse (arts. 53 y 44 ).

22. Por ejemplo, estudiante en vez de alumno/a.

23. Recordemos que en el ámbito del Ministerio de Defensa, la Orden DEF 253/2015, de 9 de febrero, modificada por la Orden DEF $112 / 2019$ establece por primera vez en la Administración Pública, la implementación progresiva del permiso de paternidad de 16 semanas.

24. Se está trabajando en un proyecto sobre la elaboración de un Curriculum Vitae anónimo. 
Tabla 1. Porcentaje de dedicación de hombres y mujeres en materia de conciliación

\begin{tabular}{ccc}
\hline $\mathbf{2 0 1 7 - 2 0 1 8}$ & \% Mujeres & \% Hombres \\
\hline Total & $\mathbf{4 8 , 4}$ & $\mathbf{5 1 , 6}$ \\
\hline $\begin{array}{c}\text { Reducción jornada por cuidado } \\
\text { de hijo menor }\end{array}$ & 70,0 & 30,0 \\
\hline $\begin{array}{c}\text { Flexibilización jornada } \\
\text { porcuidado personas mayores }\end{array}$ & 67,4 & 32,6 \\
\hline $\begin{array}{c}\text { Reducción jornada por } \\
\text { enfermedad grave de familiar }\end{array}$ & 59,4 & 40,6 \\
\hline $\begin{array}{c}\text { Excedencia para cuidado de } \\
\text { hijos y familiares }\end{array}$ & 80,0 & 20,0 \\
\hline
\end{tabular}

Fuente: Datos y cifras del Sistema Universitario Español - Publicación 2018-2019 - Ministerio de Ciencia, Innovación y Universidades.

A día de hoy, se ha comprobado que los Planes de igualdad son una medida efectiva y la propia LOIE establece su obligatoriedad en las empresas de más de doscientos cincuenta trabajadores (art. 45.2), y tienen como finalidad realizar un diagnóstico de situación, alcanzar en la empresa la igualdad de trato y de oportunidades entre mujeres y hombres y eliminar la discriminación por razón de sexo (art. 46.1)25.

Las Unidades de Igualdad tienen como misión el seguimiento y evaluación de las actuaciones establecidas en los Planes de Igualdad. En la D.A. n.12 de la LOM-LOU se establece su creación para recabar estadísticas y asesorar sobre su elaboración y realizar propuestas de mejora en acciones formativas, asesorar también sobre análisis de impacto por razón de género a la hora de elaborar normativas, y en general, revisar los planes de igualdad para ver si se están cumpliendo.

Los Informes de impacto de género son una medida creada para sensibilizar a los órganos de gobierno que aprueban las normativas, también en el ámbito universitario (art. 19 LOIE) ${ }^{26}$.

En materia de acceso a las estadísticas sobre la variable de sexo y con base en la LCTI, para el sistema de información I+D+i, existe un Sistema Integrado de Información Universitaria (SIIU) e Indicadores e informes de Ciencia, Tecnología e Innovación (ICONO) para recoger, tratar y difundir los datos desagregados por sexo. El Plan Estadístico Nacional (PEN), debe seleccionar indicadores nuevos y más precisos y dar a conocer los estudios y estadísticas en las universidades, como por ejemplo, cómo se están llevando a cabo los Planes de igualdad y qué resultados se están obteniendo.

Otros logros obtenidos se encuentran en materia de subvenciones públicas. En el ámbito universitario nacen con base en el art. 45.4 de la LOU "Becas y Ayudas al estudio", con especial atención a personas con cargas familiares, víctimas de la violencia de género (...) garantizando así su acceso y permanencia en los estudios universitarios ${ }^{27}$. Así, la Resolución de 7 de mayo de 2019 del Instituto de la Mujer y para la Igualdad de Oportunidades, por la que se convoca la concesión de las subvenciones públicas destinadas a la realización de Postgrados de Estudios de Género y Actividades del ámbito universitario relacionadas con la Igualdad de oportunidades entre mujeres y hombres para el año 2019, concede $15.000 €$ por Programa. Los premios (actos de reconocimiento) a mujeres ayudan a normalizar y difundir valores de

25. En el caso de las OPI's la LCTI en su D.A $13-6^{\circ}$ establece que se adoptarán planes de igualdad, que serán de seguimiento anual y deberán incluir medidas incentivadoras para aquellos centros que mejoren los indicadores de género.

26. Art 19 LOIE. Informes de impacto de género: "Los proyectos de disposiciones de carácter general y los planes de especial relevancia económica, social, cultural y artística que se sometan a la aprobación del Consejo de Ministros deberán incorporar un informe sobre su impacto por razón de género".

27. En esta línea, con fecha 29 de abril de 2019 se ha publicado en el Boletín Oficial del Estado (BOE n 102), el extracto de la Resolución de 17 de abril de 2019, de la Secretaría de Estado de Igualdad, por la que se convocan los Premios de la Delegación del Gobierno para la Violencia de Género a tesis doctorales sobre violencia contra la mujer para el año 2019, si contribuyen al establecimiento de medios para conocerla y evitarla. 
igualdad de oportunidades y responsabilidades ${ }^{28}$, destacando el concedido a $\mathrm{M}^{\mathrm{a}}$ Dolores Cabezudo Ibáñez ${ }^{29}$ y a Margarita Salas recientemente el pasado 20 de junio de 2019.

En esta línea, con fecha 29 de abril de 2019 se ha publicado en el Boletín Oficial del Estado (BOE n 102), el extracto de la Resolución de 17 de abril de 2019, de la Secretaría de Estado de Igualdad, por la que se convocan los Premios de la Delegación del Gobierno para la Violencia de Género a tesis doctorales sobre violencia contra la mujer para el año 2019, reconociendo y recompensando a las personas autoras de aquellas tesis doctorales que, como consecuencia de su investigación, contribuyen a mejorar el conocimiento de la dimensión, consecuencias y características de la violencia de género, así como los medios para evitarla.

Otras medidas ya existentes son la Unidad de Mujeres y Ciencia creada por el Ministerio de Ciencia, Innovación y Universidades. La Unidad de Mujeres y ciencia creada por el Ministerio de Ciencia, Innovación y Universidades que, para dar respuesta al principio de transversalidad de género con base en la normativa analizada, en los ámbitos científico y de innovación, crea planes de estudio en enseñanzas Universitarias, diseñados desde el respeto a la igualdad y creando postgrados específicos en Igualdad de Género. Procura la eliminación de sesgos, barreras y desincentivación para conseguir la presencia equilibrada de mujeres y hombres en Ciencia e Innovación; promueve la integración transversal del análisis de sexo/ género en los contenidos de programas y proyectos de investigación científica y tecnológica, así como la investigación específica en el campo de los estudios de género. Los poderes públicos deben considerar los impactos diferenciados de género y contemplar medidas para promover la igualdad entre mujeres y hombres en todos los posibles campos de desarrollo: legislación, políticas, presupuestos, planes, proyectos ${ }^{30}$

Los ya existentes "Día Internacional de la Mujer" (8 de marzo) y del "Día Internacional de la Eliminación de la Violencia contra la Mujer" (25 de noviembre).

Dentro del entonces Ministerio de Ciencia, Innovación y Universidades, se encuentra el Observatorio "Mujeres, Ciencia e Innovación para la Igualdad de Género, que se constituyó el pasado 10 de enero de 2019 por el Real Decreto 1401/2018, de 23 de noviembre y que tiene como fines: incorporar criterios sensibles al género en la formación de personal; crear un plan de formación dirigido a la alta dirección y mandos intermedios; y crear nuevas medidas de conciliación como objetivos prioritarios (art. 6). Actualmente las 6 primeras semanas son obligatorias e intransferibles para la mujer y no así las 10 siguientes. Para el hombre existe un permiso de las 5 primeras semanas voluntarias e intransferibles. Por otra parte, la Ley de Presupuestos Generales del Estado de 2019 y la Proposición de Ley en el Congreso han recogido la ampliación a 16 semanas de los permisos de paternidad y los Sindicatos reivindican una bolsa de horas de hasta un $5 \%$ de la jornada anual para el cuidado de hijos, personas mayores y discapacitados.

Entre las funciones que desempeñará el Observatorio se encuentran las de seguimiento, informe y evaluación y propuesta (art. 6), entre otras:

a. De seguimiento: recabar información sobre igualdad de oportunidades en el ámbito universitario; conocer los planes de igualdad de los OPIs y los resultados de su seguimiento anual; conocer las actuaciones realizadas por las unidades de igualdad de las universidades; el seguimiento de las actuaciones en materia de prevención y lucha contra el acoso laboral y el acoso sexual y por razón de sexo en el trabajo, incluyendo la violencia de género.

28. Por ejemplo en 2019 se han concedido 2 de tres medallas de oro a mujeres docentes e investigadoras: María Vallet Regí , Investigadora, Catedrática de Química inorgánica de la Universidad Complutense de Madrid Frase: "las mujeres eligen igual que los hombres pero las preferencias no son las mismas"

29. Catedrática, de Tecnología de los Alimentos de la Universidad de Castilla la Mancha, Profesora e investigadora del CSIC y escritora. Frase: hay que saber respetar la libertad de las mujeres. Otra frase: los actos de reconocimiento ayudan. 
b. De informe y evaluación, como evaluar el impacto, situación y actuaciones en el sistema español de ciencia, tecnología e innovación; promover estudios e informes técnicos sobre la situación de las mujeres científicas, tecnólogas e investigadoras en España.

c. De propuesta, entre las que se incluyen formular recomendaciones y propuestas para mejorar los indicadores y sistemas de información y la situación de las mujeres; proponer acciones para visibilizar la trayectoria de científicas en la historia y actuales para que sirvan de referente a las jóvenes de hoy para promover que las niñas se interesen por ramas científicas; diseñar acciones de fomento de vocaciones científicas en STEM para que las mujeres dejen de estar menos representadas.

d. En general, el Observatorio busca mejorar los criterios de la composición de las comisiones de evaluación de ayudas a la I+D+i con objeto de evitar posibles sesgos de género en sus criterios de selección; promover la igualdad de género en equipos e instituciones y poner en marcha un plan de formación dirigido a la alta dirección y mandos intermedios de los centros públicos de investigación y de sus órganos de gobierno. Buscará las claves para trabajar adecuadamente, identificar los problemas y actuar sobre ellos. Sus principales competencias consistirán en realizar un seguimiento de las actuaciones de los Departamentos Ministeriales, las Administraciones Públicas y, en general de todos los actores del sistema español de ciencia, tecnología e innovación, en materia de prevención y lucha contra el acoso laboral y el acoso sexual y por razón de sexo en el trabajo, incluyendo la violencia de género ${ }^{31}$.

e. Por otra parte, la creación de nuevas medidas de conciliación, pues el impulso de la natalidad conlleva en muchos casos apartarse temporalmente de la carrera investigadora y la reincorporación suele resultar complicada por lo que, en las convocatorias de proyectos de investigación y contratos de investigación se debe tener en cuenta el tiempo de maternidad y cuidado de los hijos pequeños (poder compatibilizar ser científico con ser padres y madres) ${ }^{32}$. Por ello considero que deben impulsarse políticas y actuaciones que consideren el tiempo de la maternidad.

\section{Nuevas medidas de acción positiva}

La introducción del género en el EEES crea un modelo de sociedad comprometida e inclusiva (DONOSO-VÁZQUEZ, 2014). Se hace relevante la puesta en marcha de nuevas medidas de acción positiva en la Universidad, entre ellas:

- Incorporar la igualdad de género en todas las Facultades para generar un cambio de mentalidad en los estudiantes porque son el futuro ejemplo para crear una sociedad de goce de derechos y libertades.

- La docencia como metodología necesita incorporar la perspectiva de género en las distintas áreas de conocimiento que promuevan la igualdad en todos los ámbitos profesionales, mediante la inclusión de planes de estudio "transversalidad" y nueva redacción de los objetivos y competencias. Por tanto, planes de estudio que contemplen transversalmente estas medidas.

- Los profesores son la clave para la fomentación en clases sobre la igualdad de género y los alumnos para la sociabilización de manera externa con su conocimiento de derechos de igualdad de género. Crear especializaciones en materia de género e impartir formación en materia de igualdad a todos los agentes universitarios (directivos, docentes, PAS y estudiantes).

- Insistir en la revisión de la cuota 40\%-60\% en puestos de Dirección y Gobierno, como establece la norma, creándose como una discriminación positiva. Representatividad de un mayor número de mujeres en la gestión y en la investigación académica. Incidir más en otras maneras más subjetivas de sensibilidad y que se consigan cumplir.

- En relación a la conciliación de la vida laboral y familiar: la corresponsabilidad es el objetivo. Para ello deben estudiarse nuevas medidas de conciliación de la vida laboral

31. Comparecencia de la Ministra Educación.

32. Disposición transitoria tercera incorporada a la Orden DEF 253/2015, de 9 de febrero, por la Orden DEF 112/2019. Implementación progresiva del permiso de paternidad de 16 semanas. "Sin perjuicio de lo dispuesto en la norma séptima del anexo 1 sobre la duración del permiso de paternidad, se tendrán en cuenta los siguientes periodos escalonados de aplicación: a) En 2019: Permiso de ocho semanas, b) En 2020: Permiso de doce semanas, cc) En 2021: Permiso de dieciséis semanas. 
y familiar como ayudas para pagar a cuidadores cuando hay que asistir a Congresos Internacionales; publicar una guía de conciliación; respetar horarios de reuniones y no ponerlos a primera hora de la mañana ni a última de la tarde; fomentar el teletrabajo; ampliar el permiso de paternidad a 16 semanas (ya en marcha) y crear guarderías en lugares de trabajo ${ }^{33}$.

- Debe hacerse más visible el trabajo que realizan las unidades de igualdad y el desarrollo realizado junto con otras instituciones para favorecer las subvenciones, ayudas, becas y premios a los que antes se ha hecho referencia.

- Así mismo, deben facilitarse medidas de conciliación, profundizar en el desarrollo estadístico, revisar las leyes educativas, trabajar de manera conjunta con otros Observatorios, dar acceso a los protocolos de violencia contra la mujer...

- Por último, trabajar con otros Observatorios de la Administración Pública, así como crear nuevos protocolos ante la violencia de género, cuestión que se analizará en el epígrafe siguiente.

Por ejemplo, con el Observatorio se pretende combatir cualquier discriminación por razón de sexo, garantizar la igualdad de oportunidades y aumentar la presencia de las mujeres en todos los ámbitos de la investigación, la innovación y la Universidad, para conseguir la igualdad en todo el sistema $1+\mathrm{D}+\mathrm{i}$.

No debe olvidarse la incorporación de nuevas medidas de sensibilización y potenciación de la transmisión de valores por parte de las instituciones encaminadas a promover la igualdad, comenzando por estudiar cómo fomentar la participación de mujeres en carreras STEM. Para ello deben estudiarse las causas que plantean la desigualdad e incluir programas de sensibilización en: a) las familias, pues el papel que desempeña la familia a la hora de asesorar sobre los distintos estudios de los hijos y los roles que han ido adquiriendo durante su infancia y adolescencia; b) escuelas, pues los valores y el proceso de orientación vocacional es también una labor de la escuela desde las primeras etapas de Educación Infantil pero principalmente en la ESO con un proceso de orientación vocacional y con la inclusión en los libros de texto de ejemplos de mujeres científicas y académicas ${ }^{34}$. También este proceso de sensibilización debe concretarse en la Formación Profesional y en el Bachillerato. En estos centros debe llevarse a cabo un proceso de orientación vocacional (tanto en humanidades, ciencias experimentales, sociales y jurídicas, o ciencias de la salud o enseñanzas técnicas -carreras STEM- ciencia, tecnología, ingeniería y matemática) adaptado a cada estudiante con sus intereses, aptitudes y valores, independientemente de que sea hombre o mujer ${ }^{35}$; c) directivos y docentes universitarios, con revisión de la ficha técnica de las materias con sus competencias, objetivos y resultados en el aprendizaje ${ }^{36} ; \mathrm{y}, \mathrm{d}$ ) los medios de comunicación social que deben dar a conocer tanto las estadísticas de éxito de las mujeres como sus logros, contribuyendo así a la eliminación de estereotipos.

Por otra parte, el Plan Estratégico de Igualdad de Oportunidades 2018-2021 (PEIO)37, tiene como objetivos: la igualdad de trato de oportunidades a través de la educación y la investigación para fomentar que las mujeres sean agentes del conocimiento, fomentando la participación de las niñas y jóvenes en STEM e incorporando criterios de género en la evaluación del rendimiento científico. El Plan se organiza en torno a cuatro grandes ejes: transversalidad de género; nuevo pacto social; ciudadanía; y, violencia contra las mujeres.

33. Disposición transitoria tercera incorporada a la Orden DEF 253/2015, de 9 de febrero, por la Orden DEF 112/2019.

34. Art. 24 f) LOIEHM “El establecimiento de medidas educativas destinadas al reconocimiento y enseñanza del papel de las mujeres en la Historia".

35. Con base en el art. 23 LOIEHM. La educación para la igualdad de mujeres y hombres: "El sistema educativo incluirá entre sus fines la educación en el respeto de los derechos y libertades fundamentales y en la igualdad de derechos y oportunidades entre mujeres y hombres. Asimismo, el sistema educativo incluirá, dentro de sus principios de calidad, la eliminación de los obstáculos que dificultan la igualdad efectiva entre mujeres y hombres y el fomento de la igualdad plena entre unas y otros".

36. Art. 24 c) LOEI La integración del estudio y aplicación del principio de igualdad en los cursos y programas para la formación inicial y permanente del profesorado.

37. Hasta la fecha de recogida de datos de este estudio se sigue aplicando el Plan Estratégico de Igualdad de Oportunidades (PEIO) 2014-2016. Objetivos citados en epígrafes 107 a 115 
Por otra parte, destacan las distintas Conferencias sobre la materia, como la Conferencia General de Política Universitaria, previstas en el art. 27 bis de la LOM-LOU”. Así mismo la Conferencia Ministerial sobre Investigación, Innovación y Educación Superior de los países del Mediterráneo en la alianza Diálogo 5+5 sobre nueva Estrategia Española de Ciencia, Tecnología e Innovación que se está diseñando para el periodo 2021-2027 y que ya será la Cuarta, por acuerdo del 10 de junio de 2019. Finalmente hay que hacer referencia a las Conferencias de Género en Europa sobre la Igualdad en la Educación Superior. Ya se han celebrado diez. La decimoprimera tendrá lugar en Madrid en $2020^{38}$.

Por último, debe señalarse que la participación activa de los Rectores en la Universidad es fundamental para ejercer una cultura de igualdad entre el hombre y la mujer, y para motivar y abrirse a que docentes accedan a Postgrados, seminarios, talleres orientados también a la igualdad de género, pues si se facilitan los recursos a todas las áreas universitarias (estudiantes, docentes, PAS y órganos de gobierno) se obtiene un resultado positivo. A finales de 2018 hubo una Cumbre de los Rectoras (CUMBRE) ${ }^{39}$ de las Universidades Públicas ${ }^{40}$ para romper el techo de cristal incorporando medidas de discriminación positiva, concluyendo en la defensa de crear un área de conocimiento de estudios de género la implantación de un Grado específico, hacer llegar al alumnado en las etapas previas para acabar con los estereotipos desde la E.S.O y la transversalidad en todos los estudios ${ }^{41}$ y conseguir incorporar hombres a Grados ocupados principalmente por mujeres y viceversa. También se concluyó que la carrera investigadora contemple periodos de maternidad y cuidado de dependientes, es decir, medidas de conciliación en la carrera investigadora, mayor esfuerzo por visibilizar mujeres investigadoras (citando sus nombres) y que aumente la dotación económica en políticas de género. Destacar que se normalice el uso del lenguaje no sexista e inclusivo, ya contemplado en el art. 18.8 LOIE, que se fomente la realización de programas de formación en liderazgo, política de "tolerancia 0" frente a la violencia machista. Por último visibilizar a las mujeres investigadoras (con nombres y apellidos) y que se fomente la formación en liderazgo.

\section{Breve análisis de la violencia de género en la Universidad}

En cuanto a medidas de acción positiva que deben contemplarse se encuentran las becas para el desarrollo de Programas de ayuda específicos para víctimas de violencia de género, para que puedan recibir la ayuda personalizada y la adaptación que en su caso fuese necesaria en el régimen docente para garantizar el acceso y permanencia a estudios universitarios, la LOIE establece que las universidades, tendrán becas, ayudas y créditos para el alumnado y modalidades de exención parcial o total del pago de los precios públicos por prestación de servicios académicos(art. 14. 6 LOIE y art. 4.7 LOMPI). En la misma línea la LOM-LOU (art. 45.4 y D.A. $4^{\mathrm{a}}$ ).

Como medidas para obtener resultados positivos entre las que ya se encuentran el Observatorio Estatal de Violencia sobre la Mujer, fortalecer el marco penal y procesal y garantizar el principio de transversalidad de las medidas, encontramos también, medidas específicas de aplicación al ámbito universitario entre las que se pueden destacar la realización de estudios sobre impacto del acoso; campañas de prevención sobre acoso y violencia en los Campus; módulos sobre violencia de género en grados y Máster; y, becas sin exigencia de rendimiento académico. Existe una Guía de prevención y atención de la violencia de género en las

38. XI Conferencia Europea sobre Igualdad de Género en la Educación Superior: CONFERENCIA 16-17-18 septiembre 2020 en ETSI Industriales -UPM-Escuela Técnica Superior de Ingenieros Industriales de Madrid, España http://upm.genderequalityconference2020.com/?lang=es

39. Debe recordarse lo que la UNESCO ha señalado en la Declaración mundial sobre la Educación Superior en el Siglo XXI: Visión y acción: cinco grandes ejes de acción: la sensibilización, el diseño curricular, la investigación y difusión, la cultura institucional y la coordinación interinstitucional. 
Universidades (Plan Nacional $(1+\mathrm{D}+\mathrm{i})^{42}$ y deben establecerse protocolos a seguir que incluyan la evaluación objetiva y confidencial, instrucción del procedimiento, emisión de informe y finalmente apertura o archivo del expediente.

En este sentido destacan: a) el Acuerdo 27/07/2011 de la Administración General del Estado que impulsa en su Protocolo de actuación; b) la Resolución de 25 de noviembre de 2015, de la Secretaría de Estado de Administraciones Públicas que aborda la movilidad de empleadas públicas víctimas de acoso; y c) el Pacto de Estado (13/5/2019) contra la violencia de género que tiene como fin principal implicar a las Unidades de Igualdad de las también de las Universidades en la realización de estudios sobre el impacto del acoso, agresiones y abusos sexuales en el ámbito universitario.

Por otra parte, como fines específicos de ámbito universitario: estudios sobre impacto del acoso, agresiones y abusos sexuales en el ámbito universitario; campañas de prevención en los Campus; módulos sobre violencia de género en Grados y Máster de profesorado no universitario; inclusión en los ciclos superiores, masters, formación especializada y líneas de asesoría y cursos personalizados del Instituto de RTVE, contenidos de especialización en comunicación y género, con especial incidencia en el tratamiento de la información relacionada con la violencia contra las mujeres.

\section{Conclusiones}

1. La Universidad es el espacio para la generación de conocimiento y constituye la base para el desarrollo de una sociedad más justa. Por ello la Universidad no cesa de descubrir nuevos retos a solventar ante las deficiencias aún existentes en materia de igualdad. Iniciativas como la celebración de Jornadas en ámbitos donde las políticas de igualdad han prosperado de manera exponencial en las últimas décadas, contribuyen a ello.

2. Mientras no haya igualdad en la responsabilidad no habrá plena igualdad entre el hombre y la mujer. La igualdad de derechos no puede llevar aparejada diferentes responsabilidades en las personas porque eso genera desigualdad de oportunidades. Una vez obtenida la igualdad formal mediante la normativa vigente, el objetivo a alcanzar a corto plazo es la igualdad real/material, al margen de las barreras voluntarias, que es una muestra que siempre hay que dejar a salvo porque hay que respetar la libertad de las personas.

3. La igualdad material debe conseguirse para los estudiantes, docentes, investigadores, PAS y órganos de dirección y gobierno. Debe incidirse más en nuevas maneras más subjetivas de sensibilidad y que se consigan cumplir, así como mejorarse los estudios estadísticos sobre igualdad y su publicidad y transparencia.

4. Las medidas de conciliación vigentes deben revisarse periódicamente sin que se creen discriminaciones por obligaciones inexcusables en el ámbito familiar, sin que el tiempo dedicado a dichas obligaciones pueda suponer de alguna manera una merma en el curriculum de la persona trabajadora y especialmente en su acceso a puestos de alta dirección.

5. Se trata de renovar la Universidad en formación transversal de género. Uno de los medios para conseguirlo ha sido la incorporación institucional de los estudios específicos en materia de igualdad en todos los ámbitos universitarios y de postgrado. Deberían también realizarse Jornadas y Seminarios en las Universidades dirigidos a estudiantes, docentes, PAS, órganos de gobierno y dirección. Las carreras en ciencia, tecnología, ingeniería y matemáticas (STEM) se consideran los empleos del futuro, ya que el Parlamento Europeo pronosticó alrededor de 7 millones de nuevos empleos STEM para 2025 solo en Europa.

6. En los Planes de Igualdad de las Universidades podría incluirse el seguimiento y evaluación de las actuaciones en Igualdad y no discriminación llevadas a cabo, como seminarios y jornadas dirigidas a estudiantes, profesorado, PAS y órganos de dirección 
donde se traslade la necesidad de garantizar el derecho a la igualdad por parte de la Universidad y los recursos que ésta pone a disposición de todos para que ello pueda hacerse efectivo; el establecimiento de un buzón y/u oficina (físico e informático) donde puedan trasladarse las quejas y sugerencias oportunas y un registro de las mismas; la revisión del protocolo de actuación frente al acoso sexual y por razón de sexo; la implementación mejorada de las políticas de lgualdad en los planes de estudio (Memorias ANECA) - de manera específica - en los objetivos, competencias, resultados de aprendizaje, etc... y - de manera transversal en todas las materias/asignaturas; la incorporación en las encuestas de calidad de la Universidad el ítem correspondiente a la satisfacción sobre la Unidad de Igualdad, como aspectos más destacados. E implantar o revisar los protocolos ya existentes de violencia contra cualquier miembro de la comunidad universitaria, ofrecer toda la información a estudiantes, docentes, PAS y directivos de manera periódica como medida de cultura y sensibilización a través de Jornadas y Seminarios.

7. Conseguir la ocupación de puestos de liderazgo por mujeres hace más visible dicha igualdad de oportunidades y el papel fundamental que las mujeres desempeñan también en el ámbito universitario favoreciendo así "cultura de la igualdad". No debe olvidarse el fomento de programas de formación en liderazgo entre las mujeres. Invertir la "brecha de género" implica una participación equilibrada.

8. Incidir en que los medios de comunicación social, que son la fuente más directa de información que tienen los jóvenes, den a conocer tanto las estadísticas de éxito de las mujeres como sus logros, lo que irá contribuyendo a la eliminación de estereotipos.

9. En definitiva la introducción de la perspectiva de género en el EEES crea un modelo de sociedad comprometida. Se hace relevante la puesta en marcha de nuevas medidas de acción positiva en la Universidad, revisar las leyes educativas, trabajar de manera conjunta con otros Observatorios, dar acceso a los protocolos de violencia contra la mujer.

10. La Universidad tiene gran responsabilidad en la aplicación de políticas de igualdad, pero no hay que olvidar que es un trabajo conjunto de todas las instituciones.

\section{Bibliografía}

AA.VV. BOSCH, E., FERRER, V. A., CAPILLA-NAVARRO, C., \& FERREIRO, V. (2011). Incorporación de los contenidos en igualdad de oportunidades y género en Grado. Universidad de las Islas Baleares.

AA.VV. DONOSO-VÁZQUEZ, T, MONTANÉ, A \& PESSOA, M.E. Género y calidad en Educación Superior. (2014). Revista Electrónica Interuniversitaria de Formación del Profesorado, 17 (3), 157-171.

AA.VV. GÁMEZ, E.; MARRERO, H. (2003). Metas y motivos en la elección de la carrera universitaria: Un estudio comparativo entre Psicología, Derecho y Biología. Anales de Psicología, 19(1), 121-131.

AA.VV. GONZÁLEZ SALA, Francisco; MORA VALERO, Belén (2014) "Características de la violencia de género en la Universidad de Valencia" Escritos de psicología, Vol.7 (2), pp.36-43.

AA.VV. HERNÁNDEZ ARMENTEROS, J.; PÉREZ GARCÍA, J. (2018). Demografía universitaria española: aproximación a su dimensión, estructura y evolución. Cuaderno de trabajo 9. Madrid: Studia XXI. Fundación Europea Sociedad y Educación.

AA.VV. LÓPEZ CUEVAS, M; DÍAZ ROSAS F (2015) "Género y liderazgo en la universidad española. Un estudio sobre la brecha de género en la gestión universitaria" Archivos Analíticos de Políticas Educativas=Education Policy Analysis Archives, Vol.23 (1).

AA.VV. MIRALLES-CARDONA C, CARDONA-MOLTÓ, M. C y CHINER, E. (2018) El compromiso académico y social a través de la investigación e innovación educativas en la Enseñanza Superior. Ed. OCTAEDRO, S.L. 
ANECA - Agencia Nacional de Evaluación de la Calidad y Acreditación

(2018) Disponible en http://www.aneca.es/Documentos-y-publicaciones/Informes-de-resultados (acceso 3 de febrero de 2019).

(2011). Protocolo de evaluación para la verificación de títulos universitarios de Grado y Máster. ANECA. Disponible en https://bit.ly/2QMkcYj (acceso 3 de febrero de 2019).

ARRANZ LOZANO, Ma Mercedes. (2001). "Hombres y mujeres en el profesorado: un análisis de género" en GARCÍA DE LEÓN, M. A. Y GARCÍA DE CORTÁZAR, M. (eds.), Las académicas (Profesorado universitario y género), pp. 337-404. Madrid. Instituto de la Mujer.

ARRANZ LOZANO, Fátima. (2004). "Las mujeres y la universidad española: estructuras de dominación y disposiciones feminizadas en el profesorado universitario". Política y sociedad, 41(2), 223-242.

BALLARÍN DOMINGO, Pilar (2015) "Los códigos de género en la Universidad". Revista Iberoamericana de Educación, Vol.68, pp.19-38.

BARRÈRE UNZUETA, Mari Ángeles,

(1997) “Discriminación, derecho antidiscriminatorio y acción positiva a favor de las mujeres". Cuadernos Civitas. Madrid.

(2001) "Problemas del Derecho Antidiscriminatorio: subordinación versus discriminación y acción positiva versus igualdad de oportunidades". Revista Vasca de Administración Pública, № 60, pp. 145 - 166.

CAPEL MARTÍNEZ, Rosa Ma. (1982) El trabajo y la educación de la mujer en España (19001930) en Madrid: Ministerio de Cultura, 1982, 363.

CARMONA-VALDÉS, Sandra Emma. (2015) Hacia una educación con equidad. Praxis, 11(1), 8-18.

COMISIÓN EUROPEA. (Ed.)

(2009) Comunicación de la Comisión al Consejo, al Parlamento Europeo y al Comité Económico y Social Europeo y al Comité de las Regiones. Comunicación de la lgualdad entre mujeres y hombres. Bruselas. Disponible en: https://eur-lex.europa.eu/ legal-content/ES/ ALL/?uri=CELEX:52009DC0077 (acceso 20 de mayo de 2020).

(2019). Actuación de la Unión Europea en favor de las mujeres. https://doi. org/10.2838/168837 (acceso 20 de mayo de 2020).

INSTITUTO EUROPEO DE LA IGUALDAD DE GÉNERO (EIGE - UE) (Ed.)

(2013a). Incorporación de la perspectiva de género en las políticas y los programas de las instituciones de la Unión Europea y los Estados miembros de la UE. Disponible en https://doi.org/10.2839/43849 (acceso 20 de mayo de 2020)

(2013b). Review of the implementation of the Beijing Platform for Action in the EU member states: Women and the media. Advancing gender equality in decision-making in media organizations. Publications office of the European Union. https://doi. org/10.2839/73389 (acceso 20 de mayo de 2020)

DECLARACIÓN DE CASTELLÓN - Disponible en https://canal.ugr.es/wp-content/uploads/2018/11/Declaracio\%CC\%81-Castello\%CC\%81-Cimera-Rectores-2018.pdf (acceso 28 de febrero de 2019)

DE GARAY, Adrián. (2013) “Igualdad de Género en la Universidad Autónoma Metropolitana: un espejismo". La ventana. Revista de Estudios de Género. Guadalajara Vol.4 n.38. Jul./Dec.

DE PABLO, Flora (2001) "Las científicas y el techo de cristal" en http: www.amit-es.org/ (acceso 13 de marzo de 2019)

DEL PINO, María (2009) “Género y Universidad” Enseñanza \& Teaching, Vol.23.

ELÓSEGUI ITXASO, María (2003). Las acciones positivas para la igualdad de oportunidades laborales entre mujeres y hombres, Ed. Universidad de Zaragoza/Centro de Estudios Políticos y Constitucionales, Madrid. 
EZQUERRA, Ramón, (1926). "La penetración de la mujer española en la Universidad. I", Renovación Social n. ${ }^{\circ} 44$ (15- VIII-1926), 490-494; Ramón Ezquerra, "La penetración de la mujer española en la Universidad. II", Renovación social n. ${ }^{\circ} 45$ (1IX-1926), 532-536; Ezguerra, Ramón."La penetración de la mujer española en la Universidad. III", Renovación Social n. ${ }^{\circ} 46$ (15-IX -1926), 629-632

FLECHA GARCÍA, Consuelo (1996). Las primeras universitarias en España: 1872-1910. Ed. Narcea. Madrid.

GIMÉNEZ-SALINAS, Esther. (2005). "Mujeres en la Universidad: cien años de prohibiciones". En: Giménez-Salinas Colomer, E. (ed.), Doctas, Doctoras y Catedráticas. Cien años de acceso libre de la mujer a la Universidad, pp. 57- 72. Disponible en: http://mujeresconciencia.com/app/ uploads/2015/02/Doctas doctoras Castellano Completo.pdf (acceso 17 de febrero de 2019)

ILO - OIT - (2009) La igualdad de género como eje del trabajo decente -. VI Informe Conferencia Internacional del Trabajo, 98.a reunión, Disponible en "https://www. ilo.org/wcmsp5/groups/public/---ed norm/---relconf/documents/meetingdocument/wcms 106175.pdf (acceso 7 de enero de 2019).

LAUROBA LACASA, María Elena, Comentario a la Ley Orgánica de Universidades . Coord. González García, Julio V. Civitas. pp. 1062-1092. (2009).

LÓPEZ-SÁEZ, Mercedes. (1995) La elección de una carrera típicamente femenina o masculina. Desde una perspectiva psicosocial: la influencia del género. Madrid, Ministerio de Educación y Ciencia.

MACÍAS JARA, María, (2011) Actas del III Congreso Universitario Nacional "Investigación y género" / coord. Vázquez Bermúdez, Isabel. pp. 1142-1164.

MANGINI, Shirley, (2001) Las modernas de Madrid. Las grandes intelectuales españolas de la vanguardia. Madrid: Península, 54.

MAINSTREAMING DE GÉNERO. (2001) Marco conceptual, metodología y presentación de buenas prácticas: Instituto de la Mujer (Ministerio de Trabajo y Asuntos Sociales), Serie Documentos, N² 28 , Madrid.

MENÉNDEZ, María Isabel. (2014). El Espacio Europeo de Educación Superior en España: Incorporación de contenidos y metodologías de género en comunicación. Revista Internacional en Comunicación y Desarrollo, 1(0), 23-34. Disponible en https://bit.ly/2t3xi9M (acceso 5 de marzo de 2019)

MILLER SALOMON, Bárbara. (1985) In the company of Educated Women. A History of Women and Higher Education in America. New Haven: Yale University Press, 141.

MINISTERIO DE CIENCIA, UNIVERSIDAD E INNOVACIÓN - Unidad de Igualdad. Disponible en http://www.ciencia "Datos y cifras - Ministerio de Ciencia.".gob.es/stfls/ MICINN/Universidades/Ficheros/Estadisticas/datos-y-cifras-SUE-2018-19.pdf (acceso hasta 23 de junio de 2019).

MINISTERIO DE DEFENSA. Centros Universitarios de la Defensa. Disponible en https:// www.tecnologiaeinnovacion.defensa.gob.es/es-es/Presentacion/Organizacion/D/ Paginas/CentrosUniversitarios.aspx (acceso 9 de enero de 2019).

MONTERO, Mercedes. (2010) Hist. Crit. No. 40, Bogotá, enero -abril.

SALDAÑA, Nieves. (2010). "Los estudios de género en los Grados en Derecho: Propuestas para un diseño curricular de la enseñanza del derecho constitucional con perspectiva de género en el Espacio Europeo de Educación Superior". Revista de Educación y Derecho, 3, 1-23. Disponible en https://bit.ly/357pMsH (acceso 5 de abril de 2019)

SIERRA HERNAIZ, Elisa. (1999) Acción positiva y empleo de la mujer, Consejo Económico y Social, Madrid.

NACIONES UNIDAS (Ed) 
(1979) Convención para la Eliminación de todas las Formas de Discriminación (CEDAW) Disponible en https://www.ohchr.org/sp/professionalinterest/pages/cedaw.aspx (acceso 9 de febrero de 2019).

(1995). Beijing Declaration and Platform for Action. Fourth World Conference on Women. Disponible en https://bit.ly/2tkGoME (acceso 7 de enero de 2019).

(2015). Transformar nuestro mundo: La Agenda 2030 para el Desarrollo Sostenible. https://bit.ly/2mej03a (acceso 20 de marzo de 2019).

UNESCO (Ed)

(1998) - Declaración mundial sobre la Educación Superior en el Siglo XXI. Revista educación superior y sociedad, 9, 2, p. 97-123.

(2012). Indicadores de género para medios de comunicación. Marco de indicadores para evaluar la sensibilidad en materia de género en las operaciones y contenidos mediáticos. Disponible en. http://bit.ly/2qWKIZN (acceso 3 de abril de 2019).

(2014). Media and gender: A scholarly agenda for the global alliance on media and gender. Disponible en https://bit.ly/2YxKSOI (acceso 4 de abril de 2019).

(2017). UnitWIN network on gender, media, and ICTs. Disponible en https://bit.ly/2KilcQ5 (acceso 4 de abril de 2019).

VERLO0, Mieke (2005). Displacement and empowerment: reflections on the concept and practice of the Council of Europe approach to gender and gender equality. Social Politics, 12(3), 344-365. Disponible en https://doi.org/10.1093/sp/jxi019 (acceso 1 de abril de 2019). 